\title{
COVID-19, cytokine storm and sigma-1 receptors: potential treatments at hand?
}

\author{
Lanfranco R. Troncone ${ }^{1}$ \\ ${ }^{1}$ Instituto Butantan
}

July 23,2020

\begin{abstract}
Fluvoxamine, a usual SSRI antidepressant, has agonistic activity on Sigma-1 receptors in ER and inhibits IRE1, an important trigger of cytokine production; Sigma-1 receptor is a target of SARS-COV-2 protein Nsp6 and may start the cytokine storm. The available Sigma-1 agonists may represent important alternatives to treat the cytokine storm and save lives.
\end{abstract}

Title: COVID-19, cytokine storm and sigma-1 receptors: potential treatments at hand?

Author: Lanfranco R. P. Troncone - PhD

Department Director

Email: lanfranco.troncone@butantan.gov.br

Affiliation:

Laboratory of Pharmacology - Instituto Butantan - São Paulo - Brazil

Av. Vital Brasil 1500 - São Paulo - Brazil

$\mathrm{PO}-05503900$

Phone: +55-11-26279763 - Laboratory - in home office now

Mobile: +55-11-999691513 - preferred

\section{Letter - Hypothesis}

As for June, 25, 2020, more than 9.5 million cases and almost 4.8 hundred thousand deaths were officially recorded worldwide. One of the most deadly clinical features of this devastating infection is the so called "cytokine storm", the uncontrolled surge in cytokines that, at a first moment is intended to fight the infection but, once out of control, starts to offend the organs and tissues of the host. Particularly in SARS-COV-2 infection, such cytokine storm has been observed after the acute infection leading to re-hospitalization with a less positive outcome. Therefore, therapeutics targeted to fight this late immune reaction of the organism may be of great benefit and life saving (Cardone et al. , 2020). In addition, most of the high throughput screening campaigns to repurpose current use drugs has focused on the anti-viral properties while the anti-cytokinestorm properties cannot be addressed in high throughput. A recent development on the therapeutic front is the demonstration that Dexamethasone can reduce the mortality rate among severe cases of COVID-19 (see https://www.recoverytrial.net/). The use of a corticosteroid to treat infectious conditions has been considered with caution given the immunosuppressive side effect of this class of drugs. Therefore, alternatives should 
always be considered. In this line, a recent paper published in late 2019, in Science Translational Medicine may bring a new alternative involving a commonly used antidepressant drug in a new function: Fluvoxamine (Rosenet al. , 2019).

One of the key modulators of cytokine secretion is the Sigma-1 receptor, an intracellular receptor with elusive and multiple functions, found in the endoplasmic reticulum of many cell types including neurons and immune cells (Schmidt et al. , 2019). Sigma-1 receptor was initially classified as opioid receptor sigma-1 but no consistent interactions with real opioids were found, so now they are better described as Sigma non-opioid intracellular receptor 1 (See IUPHAR Guide to Pharmacology). It is still considered an orphan receptor as far as an endogenous ligand is not recognized. As nicely reviewed by (Schmidtet al. , 2019), this receptor interacts with at least 49 proteins in several different ways from GPCR to ion channels and is considered a ligand-operated chaperone. What makes this mysterious intracellular receptor particularly interesting is that one of the COVID-19 proteins showed affinity for this receptor: Nsp6 (Gordon et al. , 2020). The function of Nsp6 protein is related to endoplasmic reticulum remodeling to favor Coronavirus replication. The authors above screened several Sigma1 receptor ligands for viral infectivity and/or replication and concluded that most of the antagonists had little or no effect. All the drugs tested though, were essentially antagonists of this receptor, and the aim was finding a blocking agent. The only agonist tested was dextromethorphane which presented an increase in viral titers in vitro. On the other hand, cloperastine and clemastine showed good antiviral properties and affinity for both Sigma-1 and Sigma-2 receptors but importantly, these drugs were not experimentally classified as antagonists or agonists of Sigma receptors. The authors performed a docking modeling against Sigma-1 receptor structure and observed a good fit. Clearly, the goal of this study was to identify drugs that might be repurposed to fight the SARS-COV-2 infection and replication. The question whether these drugs can control the cytokine storm, the most dangerous outcome of this infection, after pneumonia, is in focus now. In this respect a study by Rosen and colleagues(Rosen et al. , 2019) published in 2019 discovered the interplay between Sigma-1 receptors and the Inositol-Requiring Enzime 1-alpha (IRE1), an ER stress sensor. They demonstrated that both proteins are in close proximity and possibly interact. As cited by these authors, IRE1 is a potent cytokine regulator and can be activated by lipopolysacharide (LPS). They demonstrated that Sigma-1 receptors interact with IRE1 to produce hyper inflammation under the appropriate stimulation in vitro and in vivo. Also used human bone marrow derived mastocytes (BMDM) normal and KO for Sigma-1 receptors, reinforcing the translational potential of the study. The authors used a Sigma-1 receptor ligand that may represent a hope in the treatment of the cytokine storm, namely Fluvoxamine. This drug has low nanomolar affinity for Sigma-1 receptors with reported antiinflammatory effects (Ghareghani et al. , 2017). Fluvoxamine stimulated Sigma-1 receptors and effectively dampened the secretion of cytokines, increased survival rates in two in vivo models of sepsis, LPS and fecal-induced peritonitis.

As for the present state of the knowledge on the molecular mechanisms underlying the triggering of the cytokine storm, the description of one SARS-COV-2 protein with affinity for Sigma-1 receptor, which by its turn is a critical player in controlling IRE1 nuclease activity, responsible for starting the cytokine storm, may be a good clue to reach an efficient control of this devastating condition. In this respect, fluvoxamine may represent an alternative but may not be the only one. Other Sigma-1 agonists have been reported and among them clemastine, cloperastine and dextromethorphan, all readily available and in clinical use. Dextromethorphae have to be taken with caution since it may increase viral titters, as cited above but in low dose it has important protective effects in sepsis and may still be considered (Zhou et al. , 2019). Others like the PRE-084, SKF10-047 should be considered as potential game changers in the fight against this unprecedented pandemic. A careful evaluation of the evolution of infection should be made in order to make timely treatment with these potentially life saving drugs.

Cardone M, Yano M, Rosenberg AS, Puig M (2020). Lessons Learned to Date on COVID-19 Hyperinflammatory Syndrome: Considerations for Interventions to Mitigate SARS-CoV-2 Viral Infection and Detrimental Hyperinflammation. Frontiers in Immunology 11 (1131).

Ghareghani M, Zibara K, Sadeghi H, Dokoohaki S, Aryanpour R, Ghanbari A (2017). Fluvoxamine stimulates 
oligodendrogenesis of cultured neural stem cells and attenuates inflammation and demyelination in an animal model of multiple sclerosis.Sci Rep 7 (1): 4923.

Gordon DE, Jang GM, Bouhaddou M, Xu J, Obernier K, White KM, et al. (2020). A SARS-CoV-2 protein interaction map reveals targets for drug repurposing. Nature .

Rosen DA, Seki SM, Fernandez-Castaneda A, Beiter RM, Eccles JD, Woodfolk JA, et al. (2019). Modulation of the sigma-1 receptor-IRE1 pathway is beneficial in preclinical models of inflammation and sepsis. Sci Transl Med 11 (478).

Schmidt HR, Kruse AC (2019). The Molecular Function of $\sigma$ Receptors: Past, Present, and Future.Trends Pharmacol. Sci. 40 (9): 636-654.

Zhou R, Chen S-H, Li G, Chen H-L, Liu Y, Wu H-M, et al. (2019). Ultralow doses of dextromethorphan protect mice from endotoxin-induced sepsis-like hepatotoxicity. Chem.-Biol. Interact. 303: 50-56. 